\title{
Neosporosis in a white rhinoceros (Ceratotherium simum) calf
}

\author{
$\mathrm{J} \mathrm{H} \mathrm{Williams}^{\mathrm{a}}$, I Espie ${ }^{\mathrm{b}}, \mathrm{E}$ van Wilpe ${ }^{\mathrm{c}}$ and A Matthee ${ }^{\mathrm{b}}$
}

\begin{abstract}
A 16-day-old whiterhinoceroscalf died suddenly whilein excellent condition and showing no obvious previous clinical signs. It was the 9 th calf of a mature female kept freeranging with 11 other rhinoceros as well as various other game species on a 2000 hectare game breeding centre adjacent to the town of Lichtenburg and outlying cattle farmlands in the Northern Province. At post mortem examination, death was ascribed to heart failure. There was marked multifocal to coalescing subacute parasitic myocarditis with numerous protozoan bradyzoite cysts and free tachyzoites present amongst the predominantly round cell inflammatory infiltrate. The coccidian was positively identified as $\mathrm{N}$ eospora sp. using both polyclonal and murine monoclonal $\mathrm{N}$ eospora caninum antibody immunohistochemistry in the avidin-biotin technique. The parasites stained poorly with Toxoplasma gondii-specific immunoperoxidase staining. Ultrastructurally, a section of a bradyzoite-containing cyst, as well as tachyzoites, werelargely but not totally consistent with those described for $\mathrm{N}$ eospora caninum. The dam showed no sign of illness. Neosporosis affecting white rhinoceros (Ceratotherium simum) has not previously been reported. A summarised overview of neosporosis from selected publications and a recent review is given.
\end{abstract}

Key words: Ceratotherium, congenital, immunohistochemistry, myocarditis, N eospora, neosporosis, Perrisodactyla, protozoa, rhino, ultrastructure, white rhinoceros.

Williams J H, Espie I, Van Wilpe E, Matthee A Neosporosis in a white rhinoceros (Ceratotherium simum) calf. Journal of the South African Veterinary A ssociation (2002) 73(1): 38-43. Department of Pathology, Faculty of Veterinary Science, University of Pretoria, Onderstepoort, 0110 South Africa.

\section{INTRODUCTION}

Neosporosis has recently been reviewed with special reference to advances in the life cycle and biology 5 , and since the first report of the disease in $1984^{1}$, over 250 publications have appeared. An earlier review in $1996^{6}$ covered its history and biology in detail. Neosporosis has become an important multisystemic disease affecting especially cattle and dogs, and dogs have been found to shed oocysts in faeces, thus confirming the coccidian nature of the life cycle, as well as proving that dogs can serveas both intermediateand definitive hosts. $\mathrm{N}$ eospora caninum is the type species of thegenus. Other animal species in which natural infection has been reported are horses $3,7-9,15,16,18$, sheep, goats and California black-tailed deer ${ }^{5}$. Antibodies to $\mathrm{N}$. caninum have been demonstrated in sera of naturally exposed water buffalo, coyotes, red foxes and camels ${ }^{5}$, as well as in a small percentage of nondomestic captive and free-ranging felids from southern Africa, screened by the

aDepartment of Pathology, Faculty of Veterinary Science, University of Pretoria, Onderstepoort, 0110 South Africa.

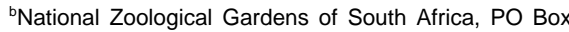
754, Pretoria, 0001 South Africa.

'EM Unit, Faculty of Veterinary Science, University of Pretoria, Onderstepoort, 0110 South Africa.

Received: May 2001. Accepted: November 2001. indirect fluorescent antibody test for both Toxoplasma gondii and $\mathrm{N}$ eospora caninum². In theseries of 68felidstested, comprising 41 lions, 4 leopardsand 23 cheetahs froma range of game reserves, national parks, 2 zoos and the De Wildt Cheetah Reserve, in South Africa as well as neighbouring Botswana and Namibia, 3 lions from the Kruger National Park and 1 cheetah from De Wildt (5.9\% of the 68 animals sampled) tested positive for both T. gondii and $\mathrm{N}$. caninum antibodies. N. caninum titres ranged from 1:50 to 1:200. None of the animals tested in the series showed any clinical signs of disease. Fifty ( $74 \%$ ) of the 68 felids were serologically positive for Toxoplasma gondii. Neosporosis has been reported in dogs and aborted twin calves in South Africa ${ }^{10-12}$, and in cattle in Zimbabwe ${ }^{13,21}$.

\section{CASE HISTORY}

A female whiterhinoceros calf was born in June 2000 to a healthy mature adult (30-35 years of age) dam that had been transported from a KwaZulu-Natal Park to Lichtenburg in Northern Province in September 1976. She had previously given birth to approximately 8 normal calves while resident at the Lichtenburg Game Breeding Centre, which belongs to the N ational Zoological Gardens of South Africa (Pretoria). This female is one of 12 free-ranging rhinoceros that share a 2000 hectare camp with other species such aswarthog, jackal, yellow mongoose, rodents, eland, springbok, impala, zebra, gemsbok, blue wildebeest, ground squirrel, porcupine, genet, springhare and scrub hare. There are no serval, caracal or hyaena (spotted or brown) in the camp. The camp comprises mainly grassland, but has a small thicket of A cacia karoo thorn-trees. It is fenced with a 1-m high diamond-mesh fence with steel wire up to $2.4 \mathrm{~m}$, topped by approximately 3 strands of barbed wire. Stones are placed along the outer base of the fence in an attempt to prevent animals from digging, but this does not stop jackal, warthog and possibly other species from crossing the boundary. The camp is surrounded on 3 sides by farmland with cattle, but there is a 5-7 strand barbed wire fence $3 m$ away from the game fence to prevent contact. One side is bordered by a wetland and a suburb of Lichtenburg. In an adjacent 2000 ha camp there are up to 25 domestic horses plus game species such as black wildebeest, tsessebe and others, with the prime aim of preventing interbreeding between dosely-related species. On occasion, the manager of the breeding centre has found and removed dogs that have managed to enter the rhinoceros camp from theadjacent suburb. Therehaveal so been occasional incidents of farmers cutting the fence outside the game fence to allow cattle access to grazing in the separating zone. There are communal water points within the camp, constructed of cement and stone, supplied by borehole water. There are also water pipelines to the town that cross the camp and occasionally develop leaks.

During an approximately 2-year period preceding thebirth of thecalf described in this case report, 2 other rhinoceros calves from different dams and both approximately 11 months of age died on separate occasions. One was too decomposed for post mortem examination by the time it was found, and the other was autopsied but no diagnosis was made.

The calf described in this report was born in the camp and wasregularly monitored after birth. It was in excellent condi- 
tion and showed no signs of illness until it was unexpectedly found dead. As soon as the mother had moved away from the dead calf, after several hours, a post mortem examination was conducted by a veterinary practitioner from Lichtenburg. Hefound a massively enlarged heart due to hypertrophy, with lesions indicating heart failure, namely generalised cyanosis with pulmonary and hepatic congestion and oedema. Samples of myocardium, liver, lung and kidney were placed in $10 \%$ formalin for histopathological examination.

\section{Microscopic findings}

Light microscopic examination of tissues stained with haematoxylin and eosin (HE), revealed marked multifocal to diffuse myocarditis with disruption and disintegration of some myofibres and separation of most myofibres by inflammatory cells consisting predominantly of macrophages interspersed with plasma cells and lymphocytes and variable numbers of neutrophils. An inciting cause was not evident with HE staining. Immunohistochemistry was undertaken using antibodies to encephalomyocarditis virus, with negative result. Acid-fast staining (Ziehl-Nielsen) and Gram staining were equally unenlightening. With Giemsastaining, protozoan-likecystsand free organisms were more easily visualised multifocally within myocardial fibres as well as between them amongst the inflammatory cells.

Immunohistochemical staining (IHC) using the avidin-biotin technique ${ }^{14}$ for Toxoplasma gondii stained the organisms only faintly compared with the known positive Toxoplasma control section. Both polyclonal and monoclonal antibodies to $\mathrm{N}$ eospora caninum stained the scattered cysts, clumps and individual organisms crisply and clearly (Figs 1, 2) like the organisms of theknown positiveN eospora control section that was stained simultaneously. The individual organisms appeared round (these probably sectioned across their short axis) to oval, with the oval parasites of the same approximate length as the diameter of the formalinfixed erythrocytes in the same sections. Cysts contained varying numbers of bradyzoites and were thin-walled (cyst wall only just visible as a thin membrane surrounding the organisms) on light microscopy.

Other HE-stained organs examined microscopically revealed mild renal congestion, marked diffuse pulmonary congestion and oedema, a few scattered intra-alveolar macrophages, as well as mild vascular neutrophilic leucostasis, and partial atelectasis of some lung

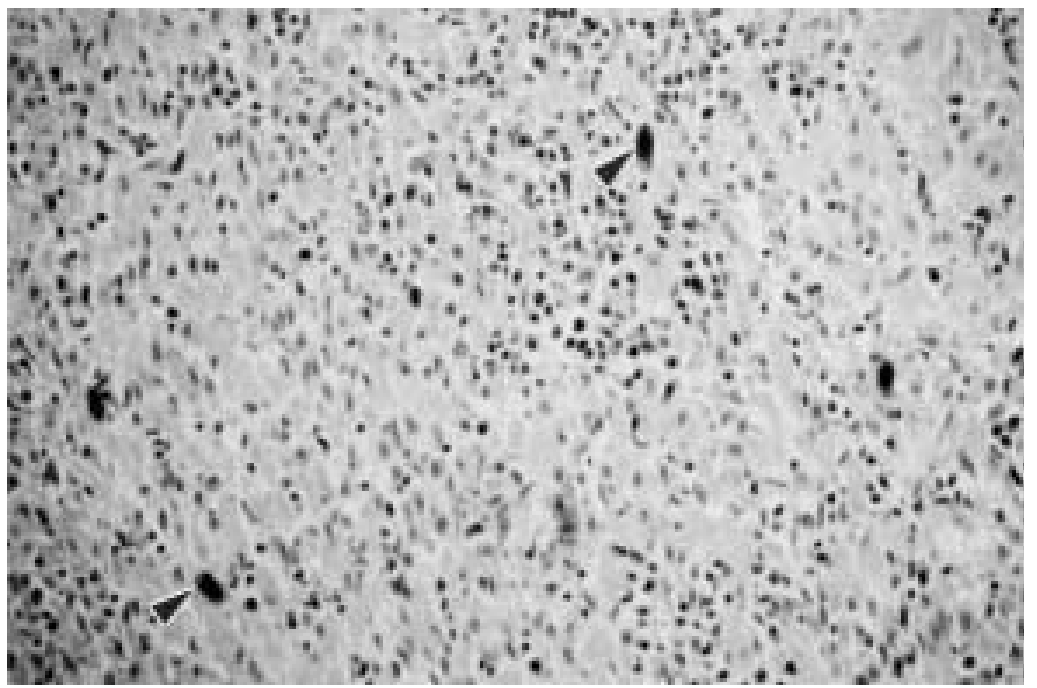

Fig. 1: Low magnification of immunohistochemically-stained section of rhino calf myocardium showing positive intra-cellular clumps/cysts of Neospora caninum (arrowheads). lobules with aeration of adjacent lobules. The liver showed marked pericentral sinusoid and central vein congestion and distension, mild centrilobular fibrosis, and either compression or acute single cell necrosis of pericentral hepatocytes, disrupting the normal hepatic lobular architecture. There was also mild to moderate vascular neutrophil and monocyte leucostasis.

\section{Ultrastructural findings}

Formalin-fixed myocardial tissue was post-fixed in $1 \%$ osmium tetroxide, rinsed in buffer, dehydrated in ethanol, and embedded in epoxy resin. Ultrathin sections were stained with uranyl acetate and lead citrateand examined in a Philips CM10 transmission electron microscope at $80 \mathrm{kV}$. Ultrastructural examination revealed intracellular tachyzoites as well as encysted bradyzoites. The tachyzoites were lying free within the host cell cytoplasm and the longitudinally sectioned ones measured $4.8 \times 0.2 \mu \mathrm{m}$. They contained a subterminal nucleus, moderately electron-dense rhoptries, micronemes, dense granules, lipid bodies, mitochondria and vesiculo-membranous organelles in their cytoplasm and they multiplied by endodyogeny (Figs 3, 4, 5).

Only cross-sections of bradyzoites were found in the ultrathin sections and these revealed dense granules, rhoptries, amylopectin granules, micronemes which in some sections were arranged perpendicular to the zoite pellicle and a nucleus within the cytoplasm. The bradyzoites were surrounded by a 0.34 $\mu \mathrm{m}$ fairly evenly-thick cyst wall that consisted of a parasitophorous vacuolar membraneand a thick underlying granular layer (Fig. 6).

\section{DISCUSSION}

The ultrastructural features of the protozoal organisms reported here were largely but not completely consistent

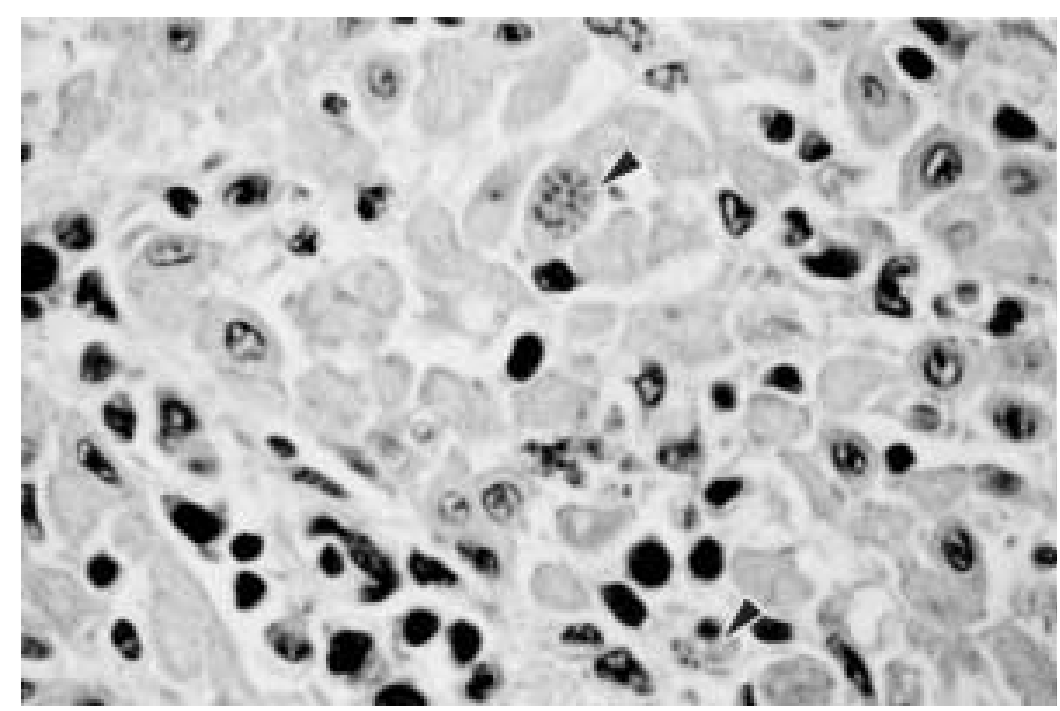

Fig. 2: High dry magnification of immunohistochemically-stained Neospora tachyzoites in the myocardium (arrowheads) with a predominantly round-cell myocarditis. 


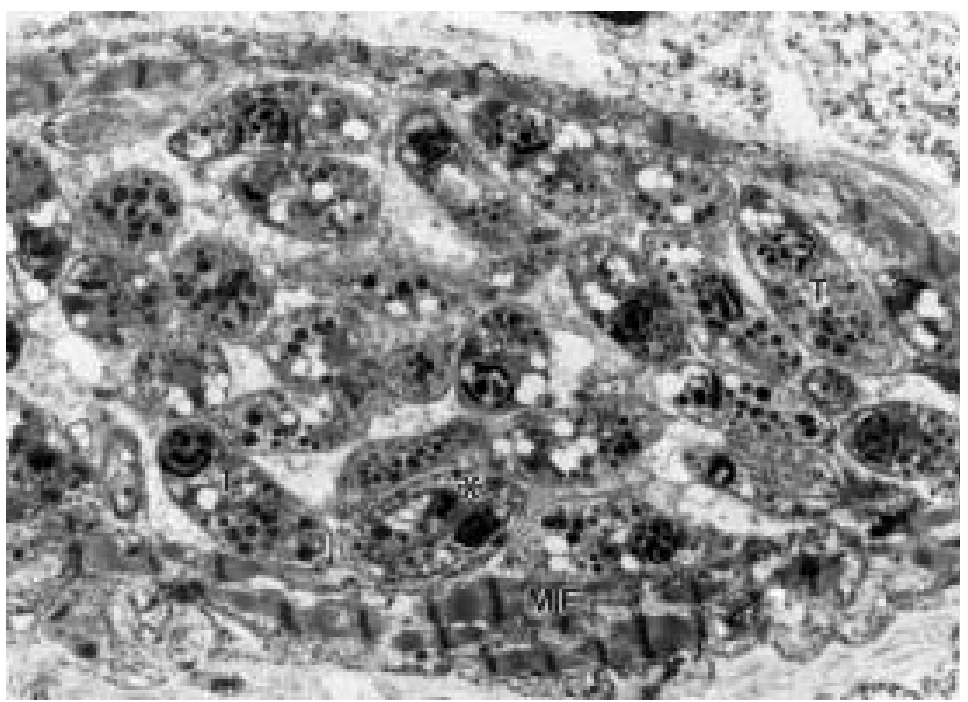

Fig. 3: Low-magnification electron micrograph of intramyocardial tachyzoites (T). Note a dividing zoite (*). MF = myofibril. $\times 8775$.

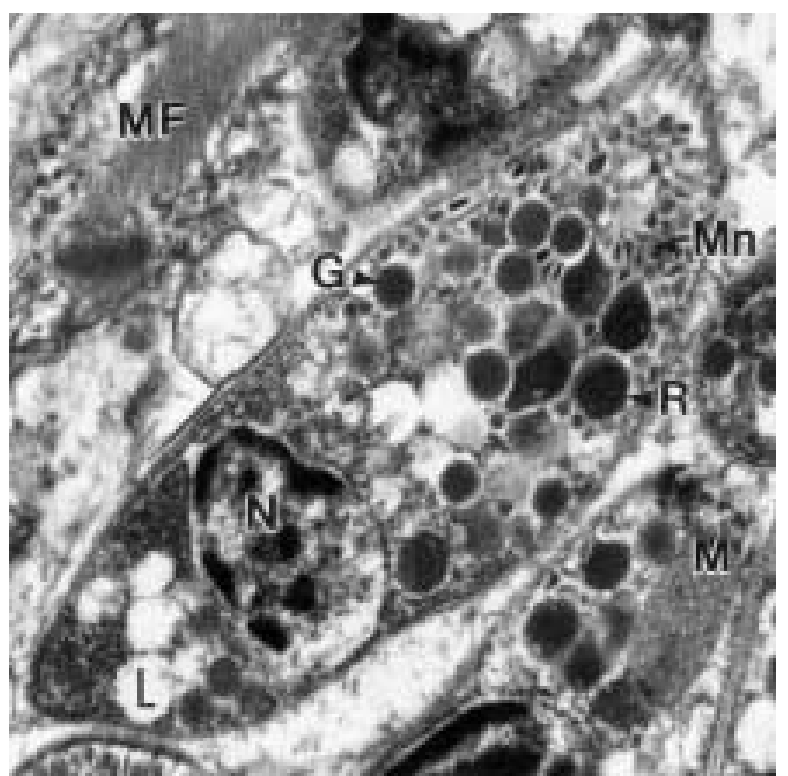

Fig. 4: Tachyzoite containing a subterminal nucleus $(\mathrm{N})$, lipid bodies $(\mathrm{L})$, dense granules $(\mathrm{G})$, rhoptries $(\mathrm{R})$, micronemes $(\mathrm{Mn})$. Mitochondria $(\mathrm{M})$ in adjacent tachyzoite. MF = myofibril. $\times 21845$.

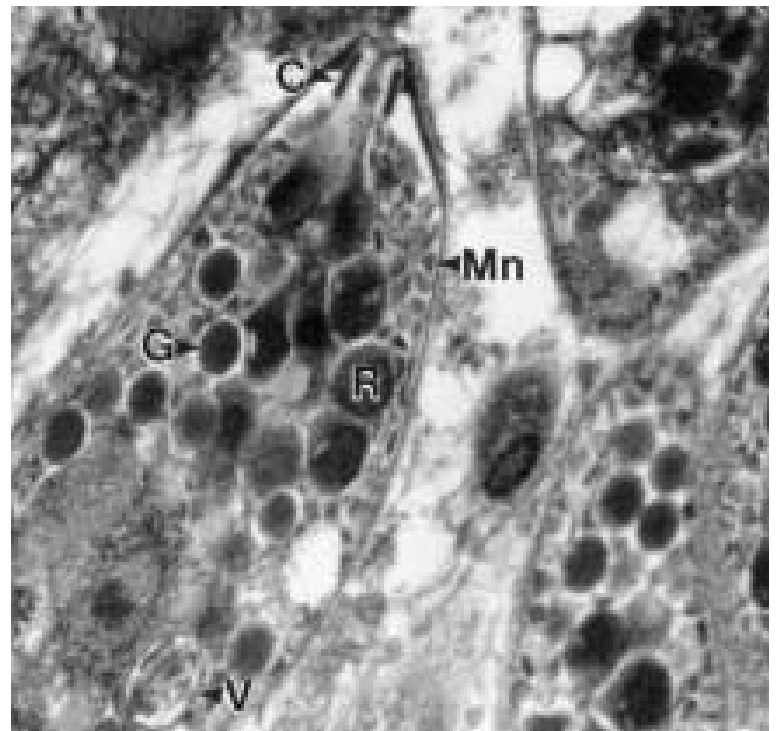

Fig. 5: Tachyzoite showing a conoid $(\mathrm{C})$ and a vesiculo-membranous organelle $(\mathrm{V})$. $\mathbf{R}=$ rhoptries, $\mathbf{G}=$ dense granules, $\mathbf{M n}=$ micronemes. $\times 23571$. with those described for $N$ eospora caninum ${ }^{19}$, showing possible overlap with Toxoplasma gondii in the smooth nature and thinness of the cyst wall and also the fact that the rhoptries were not completely electron-densealthough theinternal structure was very dark and indistinct (Fig. 7), unlike the rhoptries typical of Toxoplasma gondii which are clearly labyrinthine ${ }^{19}$. The distinct staining with polyclonal as well as monoclonal antibody to $\mathrm{N}$ eospora caninum as opposed to pale staining with Toxoplasma gondii immunohistochemistry, suggests that the parasite is a species of $\mathrm{N}$ eospora.

Rhinoceroses are related to wild and domestic Equidae, all being of the order Perissodactyla (odd-toed ungulates). The parasite in horses has recently been proposed to be another species, namely $\mathrm{N}$ eospora hughesi, based primarily on molecular differences, as well as smaller sizes of tissue cysts and bradyzoites ${ }^{17}$. Whether both $\mathrm{N}$. caninum and $\mathrm{N}$. hughesi occur in horses is unknown. A recent study in horses slaughtered in the United States showed that $21 \%$ of 296 horses had antibodies to $N$. caninum ${ }^{5}$. Possible differences in the $\mathrm{N}$ eospora spp. infecting rhinoceros could be the object of future investigation. The horses reported with neosporosis manifested clinically mostly with neurological signs and/or lesions and include an aborted foal ${ }^{7}$, a congenitally-infected female 1-month-old foal with neurological signs and blindness in both eyes ${ }^{15}$, an 11-year-old Quarter horse gelding with clinical signs of equine protozoal myeloencephalitis ${ }^{16}$ and a 20-year old horse with ataxia and antibodies to Sarcocystis neurona in cerebrospinal fluid by Western blot, but $\mathrm{N}$. caninum and not $\mathrm{S}$. neurona organisms in the CNS with IHC, light and electron microscopy 9 . A 10-year-old Appaloosa mare with chronic weight loss and anaemia that had been treated for 4 days before death with dexamethasone and penicillin was diagnosed to have visceral neosporosis when enteritis and transmural small intestinal tachyzoites as well as tachyzoites in the mesenteric lymph node were found ${ }^{8}$. One case that presented with acute neurological signs progressing to paraplegia, a 19-year-old Pinto mare with Cushing's disease dueto a pituitary adenoma, had tissue cysts and free or clustered tachyzoites in brain, spinal cord and nerve roots (cranial and sacral), but also mild multifocal nonsuppurative myocarditis with clusters of tachyzoites in myocardiocytes ${ }^{3}$. The brain of a single aborted horse foetus out of 12 equine abortions examined by PCR for $\mathrm{N}$. caninum was positive ${ }^{18}$.

Little is known about the development 


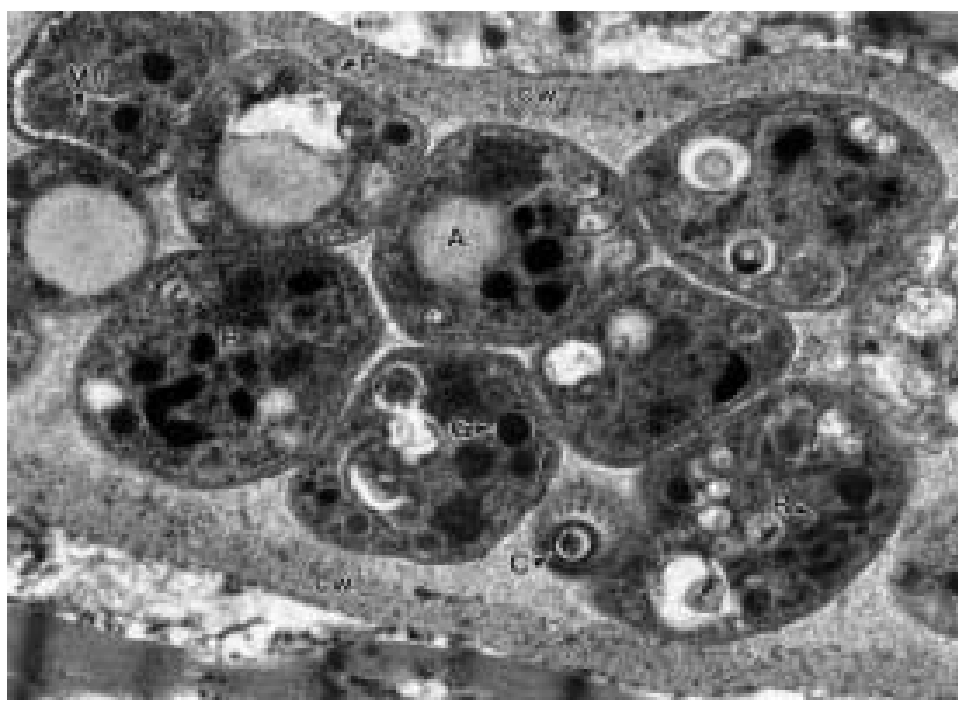

Fig 6: High-magnification electron micrograph of a portion of an intracellular cyst containing several cross-sections of bradyzoites $(B) . A=$ amylopectin granule, $\mathrm{Cw}=$ cyst wall, $\mathrm{C}=$ conoid, $\mathbf{P}=$ pellicle, $\mathbf{R}=$ rhoptries, $\mathrm{Mn}=$ perpendicular micronemes, $\mathrm{G}=$ dense granules. $\times 29242$.

and distribution in different tissues of $\mathrm{N}$. caninum in animals that are infected by natural routes (oral or transplacental). Tissue cysts were found in the brains of parenterally inoculated mice as early as 17 days post-inoculation ${ }^{5}$ and in bovine foetal brain 31 days post-inoculation of dams with tachyzoites ${ }^{5}$. Likewise, little is known of the oral infectivity of tissue cysts and tachyzoites for carnivores following ingestion or oral inoculation. It was discovered that only afew $\mathrm{N}$. caninum oocystswereshed in dog faeces, and 1 in 3 dogs shedding oocysts did not seroconvert to $\mathrm{N}$. caninum ${ }^{5}$. Nothing is known

to date regarding the frequency of shedding of oocysts, their survival in the environment, and whether canids other than domestic dogs are also definitive hosts for $N$. caninum ${ }^{5}$. Susceptible hosts may become infected by ingesting food, soil or water contaminated with $\mathrm{N}$. caninum oocystsfrom dog faeces, although this has only been shown experimentally in laboratory mice to date ${ }^{5}$. The oocysts sporulate within the intermediate host, resulting in tissuecysts containing bradyzoites. Tachyzoites are released and transmitted through the placenta to infect the foetus. Experimentally it has been shown

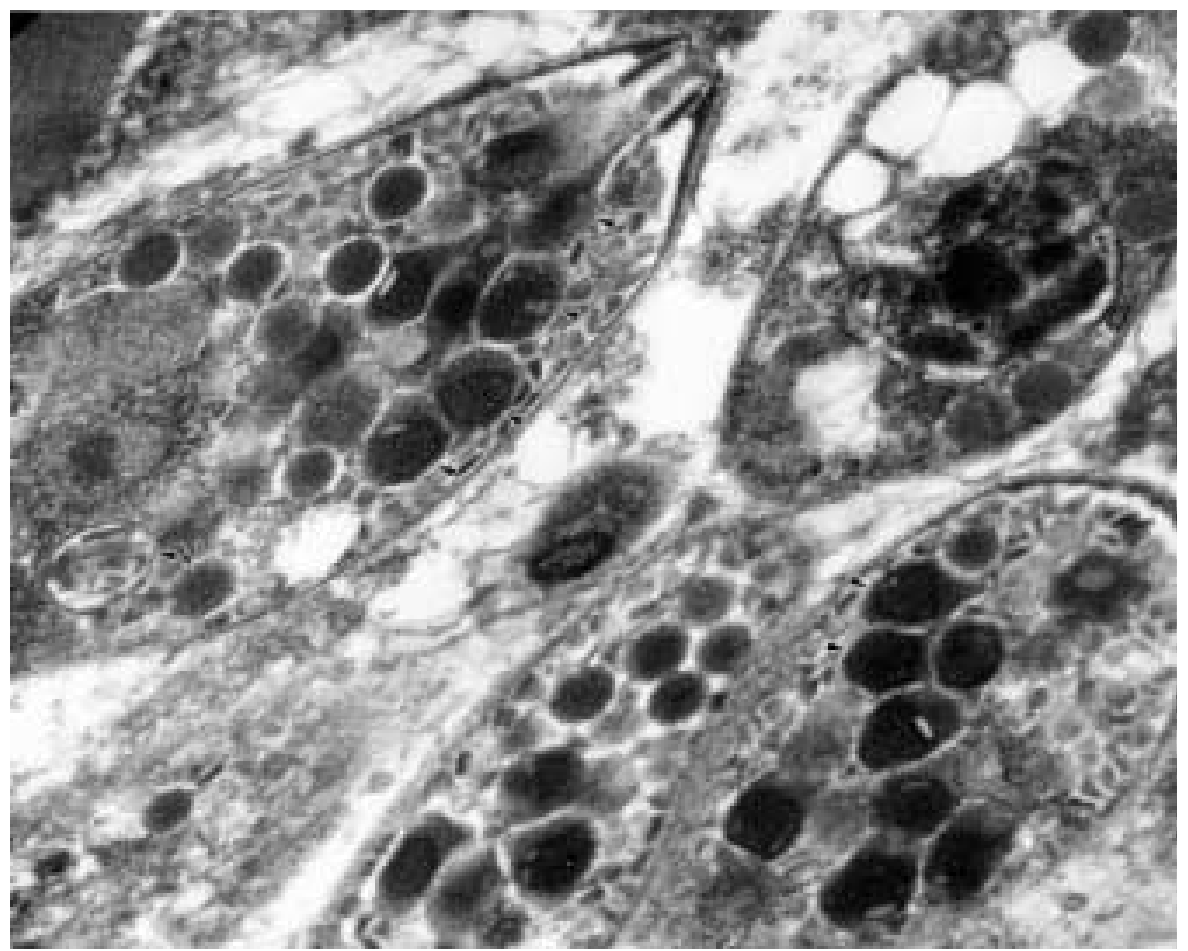

Fig. 7: Higher magnification of tachyzoite in Fig. 4 to show the internal structure of the rhoptries (arrowheads), as well as the vesiculo-membranous organelle (V). that animals may become infected lactogenically ${ }^{5}$ and calves have been infected by ingestion of milk to which tachyzoites were added ${ }^{5}$. Congenital transmission from mother to foetus has been demonstrated in cattle, sheep, goats, mice, dogs, cats, monkeys and pigs. The mechanisms of primary and repeat congenital transmission of infection areas yet unknown and it is unclear whether repeated congenital infections that occur in dogsand cattleare dueto relapse of the primary infection or to reinfection ${ }^{5}$.

When sera of dogs were screened at a 1:50 dilution in the IFAT in 4 surveys in various countries worldwide $e^{5}$, the only country where no dogs tested positive (out of 140 dogs tested), was Kenya 5 . Seroprevalences in dogs in other countries varied from $0.2 \%$ of 500 dogs in the Falkland Islands, to $29 \%$ of 194 dogs tested in Italy.

Vertical transmission of $\mathrm{N}$ eospora caninum is efficient in cattle and may proceed for several generations, but horizontal transmission appears to be necessary to introduce new infections into cattle herds ${ }^{5}$. No horizontal cow-to-cow transmission has been demonstrated in cattle. To prevent natural transmission of neosporosis from dogs to susceptible intermediate hosts, feed and water should be protected from contamination with dog faeces, and dogs should not be allowed access to aborted foetuses, foetal membranes or dead calves ${ }^{5}$. These preventative measures would not be possible in the wild, especially if wild canids were found to be definitive hosts of the parasite.

Diagnosis of exposure to $\mathrm{N}$. caninum in live animals and recently-aborted foetuses can be made by identifying the presence of specific antibodies in serum by means of several serological tests, including the ELISA, IFAT and the direct agglutination test ${ }^{5}$. Reagents for some of these tests are available commercially. An IgG avidity ELISA with the potential to discriminate between recent and chronic infections with $\mathrm{N}$. caninum in cattle has recently been described ${ }^{5}$. Examination of the foetus is necessary for a definitive diagnosis of neosporosis and ideally the whole foetus should be submitted for examination. At least samples of brain, heart and liver should be examined for histopathological changes, and body fluids or blood serum for serological evaluation. Foetal brain tissue is the most consistently affected tissue, although lesionsmay occur in several organs. There are no pathognomonic lesions, but the most characteristic finding is focal encephalitis with necrosis and non-suppurative inflammation. Hepatitis is more 
common in epidemicrather than sporadic abortions ${ }^{5}$. Most aborted foetuses autolyse rapidly, but even semi-liquid brain tissue should be fixed in $10 \%$ neutral buffered formalin for histological (HE) and immunohistochemical examination. Often only a few $\mathrm{N}$. caninum organisms are present in autolysed tissues, and these are often not visible with HE staining. Polyclonal antibodies, monoclonal antibodies and molecular biological techniques have progressively proven useful in the diagnosis of neosporosis. ${ }^{5}$. Three recombinant proteins of $\mathrm{N}$. caninum have been used for the diagnosis of bovine neosporosis. ${ }^{5}$. There is an urgent need for a commercially available $\mathrm{N}$. caninumspecific monoclonal antibody for IHC, because polyclonal antibodies sometimes cross-react with T. gondii ${ }^{6}$. Several polymerase chain reaction (PCR) methods have been reported to detect $N$. caninum $D N A^{5}$ and are still in the process of being critically evaluated in the diagnosis of $N$. caninum-induced abortion in cattle.

Clinical evidence of neosporosis in cows of any age is abortion from 3 months of gestation to term, with most occurring at 5-6 months gestation. Foetuses may die in utero, be resorbed, mummified, autolysed, stillborn, born alive but diseased, or born clinically normal but chronically infected. Abortions within a herd may be clustered, sporadic or epidemic ${ }^{5}$ and occur year-round. Serologically positive cows are more likely to abort than serologically negative cows ${ }^{5}$. Calves infected with $\mathrm{N}$ eospora caninum may be born underweight, unable to rise, and with neurological signs that may include flexion or hyper-extension of hindand/or forelimbs, ataxia, decreased patellar reflexes, loss of conscious proprioception, and exophthalmia or an asymmetrical appearance of the eyes.

Clinical neosporosis in dogs occurs most frequently in congenitally-infected young animals ${ }^{5}$, with a recently reported unusual presentation in a small number of adult dogs of nodular pyogranulomatous dermatitis ${ }^{5}$. The outstanding feature of these adult cases was severe parasitism, with large numbers of tachyzoites present. Clinical signs in young dogs are usually a progressive ascending paralysis and polyradicuIoneuritis ${ }^{4}$ ascribed to multifocal encephalomyelitis but polymyositis ${ }^{1,11,20}$ has also been reported.

Cats, mice, pigs, rats, gerbils, foxes and monkeys may be induced to be experimental intermediate hosts, in which tachyzoites and tissue cysts are the intermediate stages found, both being intracellular.

It is possible in the case reported that domestic dog faeces may have contaminated the pasture, watering points or areas around leaking pipes so that ingestion of oocysts by the dam may have occurred. Suggested modes for transmission of infection to the calf include transplacental transfer, lactogenic transfer (only proven experimentally in bovine calves so far), or possibly ingestion by the calf of oocyst-contaminated water or soil. $\mathrm{N}$ eosporosis has not yet been reported in wild canids, but since jackal belong to the family Canidaeand domestic dogs are the only currently-known definitive hosts, it is possiblethat other canidsmay al so play this role. Wild canids in the Afrotropical Region are jackals (comprising 4 Canis species), the wild dog (Lycaon pictus), the bat-eared fox (0 tocyon megalotis), and foxes (represented by 4 Vulpes species). Serological surveys of wild canid species for antibodies to $\mathrm{N}$ eospora caninum, and checking wild canids that die, especially with neurological signs, for these organisms and lesions, is important in all situations where intermediate wildlife hosts experience abortions or neonatal deaths due to neosporosis. This is relevant when considering increasingly endangered or scarce potential definitive and intermediate wildlife host species in Africa. The conservation areas that currently exist are relatively scarce and restricted in size. There is frequent overlapping of game with domestic species at the boundaries of conservation areas. Especially where disruption of fences may occur due to floods, drought, theft, war, political turmoil, ignorance, and/or poor maintenance due to economics and/or lack of manpower, these factors contributeto the increasing numbers of reports of diseases affecting both wildlife and domestic animal species. If wild canids prove to be definitive hosts of $\mathrm{N}$ eospora caninum, it is possible that they would have acquired the infection from domestic dogs or cattle (if ingestion of infected meat is found to bea routeof infection in carnivores). This, together with the fact that they are difficult to contain within fenced boundaries, may pose a threat to various potential wildlife intermediate host species, especially where cattle exist in close proximity to game, and if neosporosis is endemic in those cattle.

It is not yet known whether the dam of the calf in this report is serologically positive and was the source of infection for the apparently healthy calf, and if so, whether she will continue to produce infected or serologically positivecalves by vertical transmission, as occursin cattle. A serological survey of the cattle, domestic dogs, horses, wild canids and other potential intermediate hosts in and surrounding the Lichtenburg Game Breeding Centre might bring additional information to light with regard to this emerging and as yet enigmatic disease, of which there have been few reports in Africa.

\section{ACKNOWLEDGEMENTS}

Dr L Schaap, general practitioner in Lichtenburg, who conducted the post mortem examination on the rhinoceros calf and took the specimens, is gratefully acknowledged for his contribution and submission of tissuespecimens. Wethank Mrs Marie Smit, Mrs Anna Weldhagen, MsJoey Breedtand Mr Peter Mokonoto of the Pathology Laboratory of the Faculty of Veterinary Science at Onderstepoort for the preparation and staining of the sections, and the late Miss C Botha of the Photography Section for the preparation of the photographs.

\section{REFERENCES}

1. BjerkåsI, Mohn SF, Presthus] 1984Unidentified cyst-forming sporozoan causing encephalomyelitis and myositis in dogs. Zeitschrift für Parasitenkunde 70: 271-274

2. Cheadle M A, Spencer J A, Blagburn B L 1999 Seroprevalences of $N$ eospora caninum and Toxoplasma gon dii in nondomestic felids from Southern Africa. Journal of Z00 and Wildlife M edicine 30: 248-253

3. Daft B M, Barr B C, Collins N, Sverlow K 1996 N eospora encephalomyelitis and polyradiculoneuritis in an aged mare with Cushing's disease. Equine Veterinary Journal 29: 240-243

4. Dubey J P $1990 \mathrm{~N}$ eospora caninum: a look at a new Toxoplasma-like parasite of dogs and other animals. Compendium for the Continuing Education of Practising Veterinarians 12: 653-663

5. Dubey J P 1999Recent advances in N eospora and neosporosis. Veterinary Parasitology 84: 349-367

6. Dubey J P, Lindsay D S 1996 A review of N eospora caninum and neosporosis. Veterinary Parasitology 67: 1-59

7. Dubey J P, Portefield M L 1990 N eospora caninum (Apicomplexa) in an aborted equine fetus. Journal of Parasitology 76: 732-734

8. Gray M L, Harmon B G, Sales L, Dubey J P 1996 Visceral neosporosis in a 10-year-old horse. J ournal of Veterinary D iagnostic Investigation 8: 130-133

9. Hamir A N, Tornquist S J, Gerros T C, Topper M J, Dubey J P 1998 N eospora caninum-associated equine protozoal myeloencephalitis. Veterinary Parasitology 79: 269-274

10. Jacobsen L S, Jardine J E 1993 N eospora caninum infection in three labrador littermates. Journal of the South A frican Veterinary Association 64: 47-51

11. Jardine J E, Dubey J P 1992 Canine neosporosis in South Africa. Veterinary Parasitology 44: 291-294

12. Jardine J E, Last R D 1993 N eospora caninum in aborted twin calves. Journal of the South African Veterinary Association 64: 101-102

13. Jardine J E, Wells B H 1995 Bovine neosporosis in Zimbabwe. Veterinary 
Record 137: 223

14. Lindsay D S, Dubey J P 1989 Immunohistochemical diagnosis of $\mathrm{N}$ eospora caninum in tissue sections. A merican Journa of Veterinary R esearch 50: 1981-1983

15. Lindsay D S, Steinberg $H$, Dubielzig R R Semrad S D, Konkle D M, Miller P E, Blagburn B L 1996 Central nervous system neosporosis in a foal. Journal of Veterinary Diagnostic Investigation 8: 507-510

16. Marsh A E, Barr B L, Madigan J, Lakritz J, Nordhausen R, Conrad P A 1996 Neosporosis as a cause of equine protozoal myeloencephalitis. Journal of the American Veterinary M edical Association 209: 19071913

17. Marsh A E, Barr B C, Packham A E, Conrad P A 1998 Description of a new Neospora species (Protozoa: Apicomplexa: Sarcocystidae). Journal of Parasitology 84: 983-991

18. Pronost S, Pitel P H, Romands S, Thulliez $\mathrm{P}$, Collobert C, Fortner G 1999 N eospora caninum: first case in France in an aborted equine fetus. Pratique Veterinaire Equine 31: 111-114

19. Speer C A, Dubey J P, McAllister M M, Blixt
I A 1999 Comparative ultrastructure of tachyzoites, bradyzoites, and tissue cysts of N eospora caninum and Toxoplasma gondii. International Journal for Parasitology 29 : 1509-1519

20. Weissenbock H, Dubey J P, Suchy A, Sturm E 1997 Neosporosis causing encephalomalacia and myocarditis in young dogs. Wiener Tieraztliche $\mathrm{M}$ onatsschrift 84 233-237

21. Wells B H 1996 N eospora-induced abortion in a dairy herd in Zimbabwe. Zimbabwe Veterinary Journal 27: 9-11, 13-15

\title{
Book review - Boekresensie
}

\section{Alternative and complementary veterinary medicine}

\author{
Are Simeon Thoresen
}

\begin{abstract}
2001. A re Thoresen, Leikvollgata 31, N 3213 Sandefjord, N orway, hard cover, 544 pp., price on request from arethore@ online.no, http://home.online.no/ arethor - ISBN 8299417228
\end{abstract}

This book is the 2nd edition of the original written in Norwegian. It has been translated into English by Phil Rogers MRCVS, one of Thoresen's students from Ireland. AreSimeon Thoresen graduated from theOslo Veterinary School in 1979. Thereafter, he studied various aspects of alternative and complementary medicine, including homeopathy, acupuncture and anthroposophical medicine in various European countries as well as the USA. He uses holistic methods in his own practice. He also treats human patients holistically and gives lectures and courses on the use of complementary treatment methods.

The aim of the book is to give both professional and lay readers an understanding of the philosophy, principles and practice of holistic medicine. It is not intended as a textbook, but should rather beconsidered an introductory text on holistic concepts of diagnosis and therapy. Rather than advocating the use of alternative therapeutic modalities above conventional ones, the author sees conventional and alternative remedies as complementing each other in a holistic approach to health and disease. He encourageshisreadersto think abouthealth and diseasein a holistic way and emphasises the importance of discovering the root cause of a disease. The fundamental concept that is put forward throughout the book is that root causes of disease can be traced to imbalances of Vital Energetic Processes in the body that are precipitated by External or Internal Stressors and then manifest as Lesion-Symptom Complexes in Weak Structures of an animal/human.

The book is logically structured, with the first 3 chapters introducing the basic concepts and philosophy of holistic and complementary medicine and methods of diagnosis and therapy. The next 3 chapters are dedicated to the individual therapeutic modalities of acupuncture, homeopathy and herbal therapies respectively. Neural therapy, osteopathy, chiropractic and anthroposophical therapy are discussed together in the following chapter. Theoretical and practical thought processes are discussed followed by 'recipes' and practical treatment suggestions for specific conditions. Practical examples are interspersed in the text asillustrations of theapplication and efficacy of each treatment modality. A chapter that discusses the practical implementation of holistic methods in veterinary medicineis also included. Questions of how both veterinarians and non-veterinarians should go about starting to use alternative therapeutic methods and which conditions are best suited to this approach are addressed. The final chapter discusses the importance of nutrition, environment and biorhythmsin diseaseprevention. Onceagain, emphasisis placed on energiesand thestimulation or balancing of body Processes.

The ideasexpressed arevery different from conventional medical thinking. Methods of therapy are seen as regulating and stimulating Vital Energetic Processes. Even the effect of herbal therapies is seen to be dueto holistic stimuIation of autoregulation mechanisms of the body rather than dueto specific activecompoundsin theplant, asisthe opinion of conventional thinkers. Theauthor seesthecombination of conventional medicine with complementary treatment as being appropriatein a holisticapproach to the treatment of disease since the complementary treatment stimulates theanimal'svital energies and the conventional treatment can help to alleviate the patient's symptoms without interfering with the efficacy of the complementary treatment.

The book presents a comprehensive overview of the differentalternativeand complementary therapies used in veterinary medicine as well as the philosophy of holistic diagnosis and therapeutics. The format and writing style makes the book easy and enjoyable to read. Arguments are, however, not supported scientifically. Critical, conventionally trained readers may be disappointed and will not accept many of thestatementsmade. The book can be useful for veterinarians interested in applying alternative methods in their veterinary practice, but could also be interesting reading for sceptics who would like to find out moreabout the holistic approach to treatment and prevention of disease.

D van der Merwe ARC-Onderstepoort Veterinary Institute Onderstepoort

R Gehring

Faculty of Veterinary Science University of Pretoria Onderstepoort 